\title{
Survey of channel assignment algorithms for multi-radio multi-channel wireless mesh networks
}

\begin{abstract}
Over the past few years, the wireless mesh network (WMN) with a multi-radio multi-channel (MR-MC) has attracted increasingly high attention because of its wider coverage area. The use of multiple radios and the function of multi-hop forwarding allows WMN to achieve a greater capacity and coverage area. MR-MC can be used to utilize the radio spectrum efficiently. However, the performance of WMN is highly affected by several radios operating at frequencies close to each other. This problem can be solved using one of the key techniques called channel assignment (CA). In this paper, we first present the six main constraints of CA algorithms, i.e., interference, delay, routing, connectivity, congestion, and link scheduling. Then, various CA techniques proposed in the literature to improve the performance of WMN are discussed in detail.
\end{abstract}

Keyword: Channel assignment; IEEE 802.11; Multi-radio multi-channel; Wireless mesh networks 